Case

Report

\title{
Early Surgical Reconstruction of Sternum with Longitudinal Rigid Polymer Plating after Acute Chest Trauma
}

\author{
Jeko M. Madjarov, MD, ${ }^{1}$ Michael G. Katz, MD, PhD, ${ }^{2}$ Peter N. Kane, MD, ${ }^{3}$ \\ Svetozar Madzharov, MD, ${ }^{1}$ and Francis Robicsek, MD $^{1}$
}

\begin{abstract}
Purpose: The surgical management of the patients with traumatic sternal fractures remains controversial. The aim of this study was to evaluate the effectiveness of an early surgical reconstruction of a displaced sternal fracture utilizing longitudinal rigid polymer fixation in the settings of an acute chest trauma.

Methods: To perform the sternal fixation, we utilized a longitudinal rigid plating system. The plate is made of polyether ether ketone (PEEK), an organic thermoplastic polymer. Results: We used the entire length of the plate on each side of the fracture, secured in multiple places with 6-8 screws. Once the plates have been fully secured we tighten all the screws with a screwdriver. We demonstrated that the method minimizes pain and prevents the development of pulmonary complications.

Conclusion: This technique provides cosmetically acceptable results, minimizing risk of sternal nonunion, and decreases length of hospitalization.
\end{abstract}

Keywords: chest trauma, longitudinal rigid fixation, sternal fracture

\section{Introduction}

Chest trauma with sternal fracture is a significant source of morbidity and mortality in North America. The emergency workup of a patient with chest trauma is usually performed by cardiothoracic surgeon. A majority of cardiothoracic surgeons considered themselves competent

${ }^{1}$ Department of Cardiovascular Surgery, Sanger Heart \& Vascular Institute, Charlotte, NC, USA

${ }^{2}$ Cardiovascular Research Center, Department of Cardiology, Icahn School of Medicine at Mount Sinai, New York, NY, USA

${ }^{3}$ Department of Cardiovascular Surgery, New Hanover Regional Medical Center, Wilmington, NC, USA

Received: August 28, 2017; Accepted: November 13, 2017

Corresponding author: Michael G. Katz, MD. Icahn School of Medicine at Mount Sinai, One Gustave Levy Place, New York, NY 10029, USA

Email: Michael.Katz1@mssm.edu

(C)2018 The Editorial Committee of Annals of Thoracic and Cardiovascular Surgery. All rights reserved.

This paper was presented at the Chest Wall Injury Summit, Park City, Utah, USA, March 4-5, 2017. to perform sternal fracture repair. ${ }^{1)}$ However, the optimal surgical management of these patients, indications and timing of intervention are not well delineated and remain controversial. ${ }^{2)}$ Conservative therapy for sternal fractures with displacement, frequently fails and leads to a sternal nonunion with development of a false joint or overlap deformities. Predisposing factors for delayed or impaired sternal union include advanced age, osteoporosis, diabetes, and corticosteroid therapy. Contributing factors include poor bone-to-bone contact, hematoma formation, intravenous drug abuse, and a staphylococcal infection source. Sometimes isolated sternal fractures cause osteomyelitis, sternal abscess, and mediastinitis. Other complications following traumatic sternal fractures with displacement include persistent chest pain, with a mean duration of 8-12 weeks. Quite often the pain leads to respiratory derangement and potential lung and chest infection. ${ }^{3)}$ Several surgical techniques for sternal fixation have been described such as wire cerclage, metal osteosynthesis, nylon bands, mersilene tape, and locking plates. All approaches, however, have several limitations. ${ }^{4)}$ 


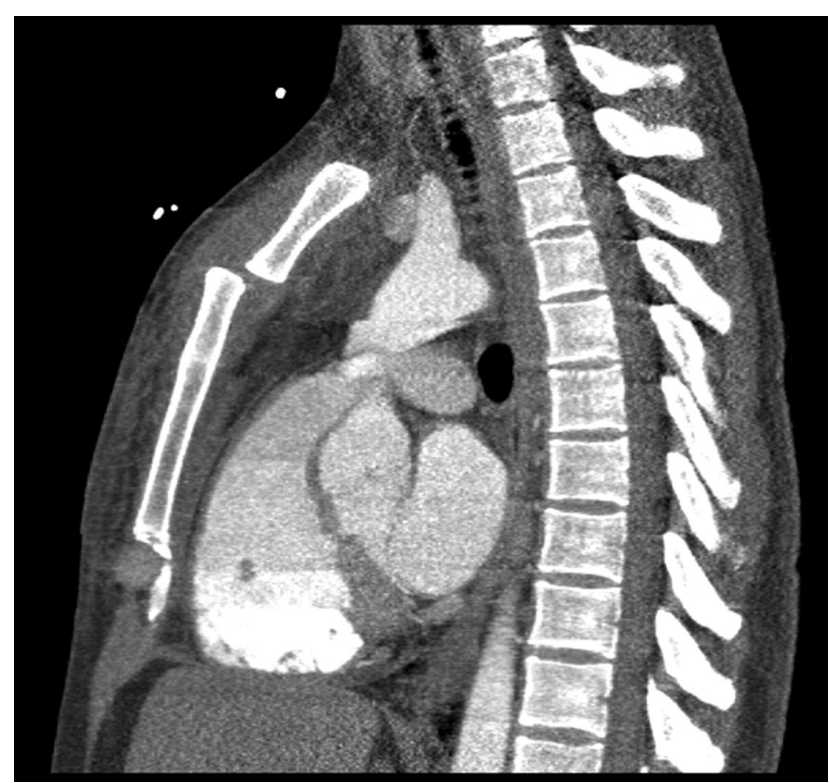

Fig. 1 Contrast-enhanced computed tomography sagittal image demonstrates manubrial-sternal dislocation.

Recently, the use of rigid metal plate fixation in sternal dehiscence and mediastinitis after cardiac surgery has been proposed with promising results. ${ }^{5)}$ This technique can be used safely and effectively in poststernotomy patients as prophylaxis against the development of sternal dehiscence and/or mediastinitis in high-risk patients with obesity, severe osteoporosis, chronic obstructive pulmonary disease, and immunocompromised state. ${ }^{6}$ The aim of this study was to evaluate the effectiveness of an early surgical reconstruction of a displaced sternal fracture utilizing longitudinal rigid polymer fixation in the settings of an acute chest trauma.

\section{Case 1}

A 26-year-old man presented to our facility complaining of persistent intractable retrosternal pain during inspiration, dyspnea on exertion, and pain when lifting his arms or rotating his body. The symptoms began 2 days prior after a high-speed motor vehicle accident during an auto race. He was taken to a local emergency room but was not hospitalized. During the first 48 hours, he suffered from intractable pain, which required high doses of narcotics. The initial medical treatment resulted in short-term alleviation of the symptoms. However, after few hours, the pain returned and the patient was presented to our institution for evaluation and treatment.

On examination, pulse oximetry revealed an oxygen saturation of $94 \%$ on room air. The sternum was painful

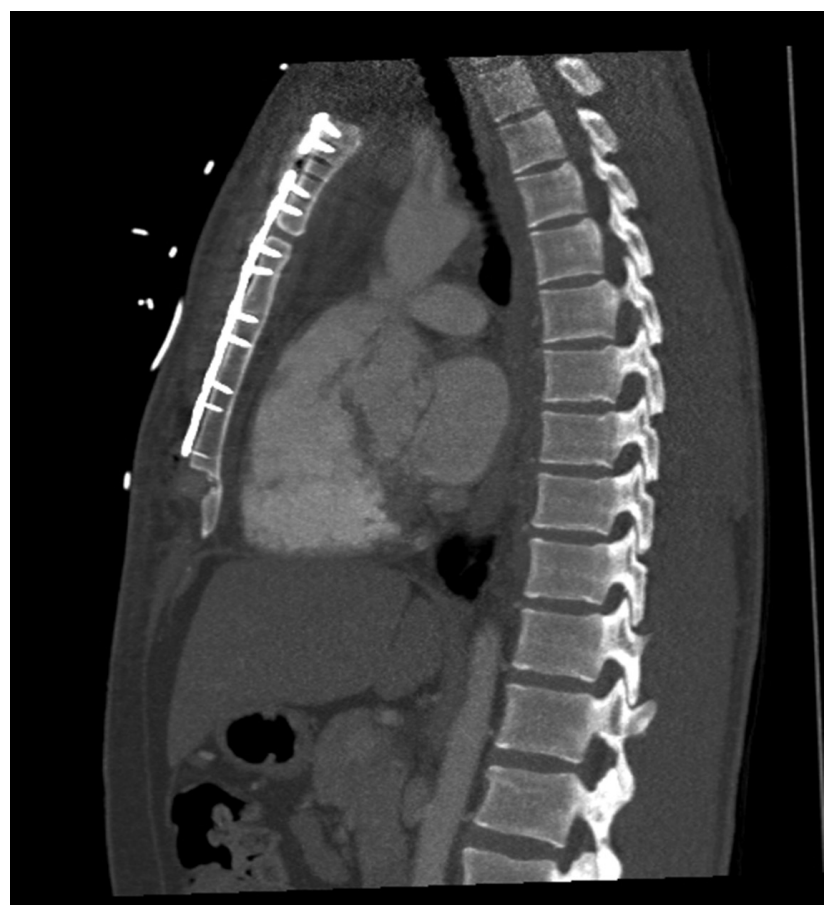

Fig. 2 Postoperative contrast-enhanced computed tomography sagittal image after surgical reconstruction.

to palpation. The lungs were bilaterally clear to auscultation. Computed tomography (CT) scan showed manubrialsternal dislocation, no evidence of a pneumothorax, or pericardial/pleural effusion (Fig. 1). Chest CT angiography confirmed the diagnosis of sternal fracture.

In view of his symptomatic sternal fracture, the patient was offered surgical treatment. The following day the patient underwent sternal reconstruction with longitudinal rigid fixation. The procedure lasted $40 \mathrm{~min}$ and went well. After extubation in the operating room, the patient progressed well, with decreased narcotic requirements and improved incentive spirometry parameters. Postoperative chest $\mathrm{X}$-ray was stable with no acute findings and excellent reduction of the displaced sternal fracture. The patient was discharged home on postop day 1 and returned to work in 6 days. The 3-month follow-up was uneventful with stable CT scan findings (Fig. 2). At long-term follow-up 39 months later, the patient reported an excellent clinical result, unrestricted range of motion without pain or functional deficit.

\section{Case 2}

A 29-year-old active and healthy male had a surfing accident that resulted in an acute chest trauma. The patient was admitted to the hospital with instantaneous 
severe pain and difficulty in breathing. In the emergency room, both chest X-ray and CT scan revealed a transverse sternal fracture in the upper part with posterior displacement. The vital signs were stable. The patient could not take a deep breath due to the severe chest pain with inspiration or even minor upper body maneuvers. The assessed pain score using the numeric pain rating was 8 of 10 . We used the verbal numeric pain scale. ${ }^{7)}$ The patient was taken directly to surgery that same afternoon where rigid longitudinal fixation of the fracture was performed. In the recovery unit just 3 hours after surgery, the patient described a relief of his severe preoperative pain and was able to take deep breaths without difficulty. The following morning the patient was ambulating and was discharged home. The patient returned to work within a week. Three-month follow-up radiographs revealed good fracture healing with excellent bone approximation. On 14-month follow-up, the patient was doing very well with excellent clinical evidence of sternal healing and no limitations in physical performance.

\section{Technique}

To perform the rigid sternal fixation, we utilized a KLS plating system. The plate is made of polyether ether ketone (PEEK), an organic thermoplastic polymer (KLS Martin, FL, USA).

Under general anesthesia with endotracheal intubation, the patient was placed in the supine position with both arms tucked along the sides. The sternum was exposed through a midline incision. In the first case, the entire length of the incision was around $8 \mathrm{~cm}$ and in the second case $\sim 12 \mathrm{~cm}$. The incision was then deepened with electrocautery. Soft tissue overlying the sternum was mobilized to expose the entire length of the bone. Preserving the priosteum, the medial attachments of the pectoralis major muscles were partially detached bilaterally and mobilized via gentle retraction. Partial pectoralis flaps were created exposing the lateral edges of the sternum. It is important to expose as much sternum as possible to disperse the forces that may ultimately disrupt a good repair. Once the bone is exposed, we used penetrating towel clamps to reduce the fracture and bring the sternal edges together appropriately. Lateral exposure of the sternum facilitates proper application of the bone reduction clamps and calipers. This allows for an accurate measurement of bone depth.

The thickness of the sternum was also measured from preoperative radiographic studies: CT scan and lateral chest X-ray. We used a power screw driver which locks

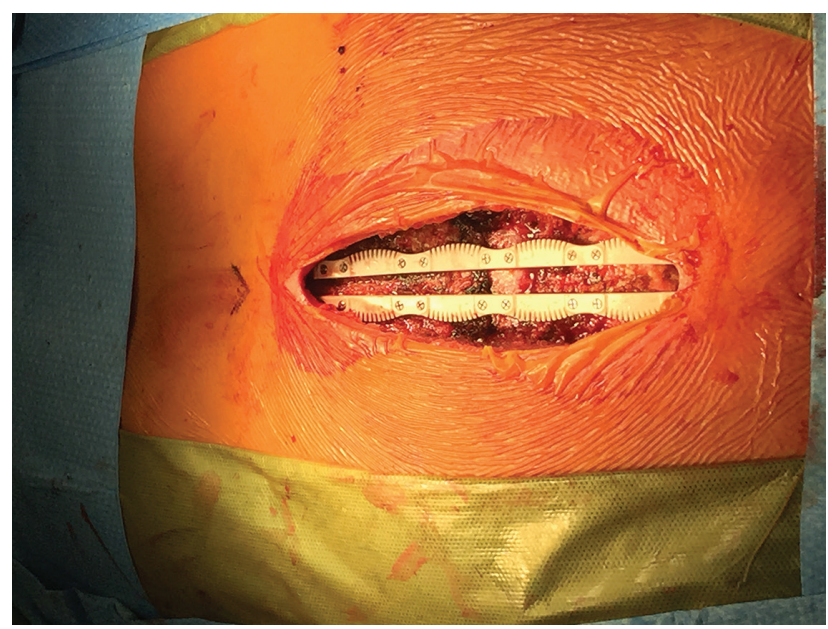

Fig. 3 An intraoperative image of surgical reconstruction with longitudinal plating.

the screws into the plate. The length of the screws is determined by the thickness of the bone plus $2 \mathrm{~mm}$ to account for the profile of the plate. The periosteum is left intact. Usually, we used the entire length of the plate (14-20 cm), secured in multiple places (requiring 6-12 screws for each plate). Our goal was to support the sternum in its entire length from the sternal notch to the level of the xyphoid process.

Two plates were used, one on each side of the fracture. The transverse fractures were reduced with the plate being fixed proximally and applying gentle manual adjustments without the need for any special equipment (in one case, we used a bone reduction clamp). Once the plates have been fully secured, we tighten all the screws with a manual screwdriver (Fig. 3).

After confirming the stability of the repair by manual palpation, a polydioxanone suture (PDS) is utilized to close the pectoralis muscle over the hardware. A routine multilayer soft tissue closure is then used to complete the procedure. No drains are required.

\section{Discussion}

\section{Potential indications for early surgical reconstruction with longitudinal rigid plating after sternal displacement}

All sternal fractures must be assessed and evaluated carefully by cardiothoracic surgeon at the time of initial presentation in the emergency department. The optimal timing of fixation of acutely displaced sternal fractures is not defined in the literature. ${ }^{1)}$ The majority of the reports describe surgical repair for sternal nonunion not earlier 
than 6-8 weeks after the initial trauma. ${ }^{1)}$ In our point of view, in the acute period of chest trauma, surgical correction is potentially easier and faster because of the absence of local inflammatory processes and overlap bone deformities. We believe that many of the chronic patients with sternal fractures will benefit from early surgical correction. Indications for that include i) cessation of intractable severe chest pain that can seriously compromise respiratory mechanics and exacerbate underlying lung injury and avoiding many adverse effects of opiates such as respiratory depression, gastrointestinal problems, dependency, etc., ii) early re-establishment of the proper orientation of the sternum can prevent cosmetic deformity of the chest with future formation of a false joint. Moreover, we conclude that after surgical repair, such patients may potentially return to regular physical activity much sooner than after conservative treatment.

\section{Preoperative planning}

The plain chest X-ray is useful to show the level of the fracture, and CT scan with three-dimensional reconstruction is helpful for assessing the position of the fragments and the degree of overlap. This also helps to rule out other significant mediastinal injuries.

\section{Comparing different surgical techniques}

The sternum is a dynamic structure that experiences several forces in multiple dimensions during respiration and activity. Because of that, steel wire fixation which is less expensive and widely used for displaced sternal fractures, often fails in transverse fractures in acute and chronic settings. Compared to the wire closure and/or rigid fixation with "short" metal plate, longitudinal fixation using a PEEK plate provides better support along the whole length of the sternum with the potential for less pain and faster healing. Current studies suggest that rigid longitudinal fixation with polymer plates significantly improves the biomechanical stability of the sternum through the enhanced resistance from transverse and longitudinal shear forces compared to regular wire cerclage. ${ }^{8)}$ In our experience, this repair is a reproducible procedure, stabilizing the whole length of the sternum with minimal expose of costal cartilage and minimal risk of iatrogenic injuries. If the sternal surface is not smooth, the polymer plate can be easily "remodeled" to align to the contour of the sternum due to the special design and material.

In addition, the surgical incision is cosmetically acceptable. The technique of longitudinal rigid sternal fixation can potentially be cost-effective, considering shortening the hospitalization and overall improvement in the postoperative course leading to quick return to normal function and activity for the patient.

\section{Conclusion}

Early surgical reconstruction with longitudinal rigid fixation with plates and screws can be successfully used after acute blunt chest trauma with sternal displacement. This technique in our reported cases minimizes pain and potentially prevents the development of pulmonary complications. It also provides cosmetically acceptable results, with faster healing. The technique could facilitate future sternotomy if clinically indicated.

\section{Funding}

This work was supported by Heineman Foundation, NY, USA

\section{Disclosure Statement}

All authors have no conflict of interest.

\section{References}

1) Mayberry JC, Ham LB, Schipper PH, et al. Surveyed opinion of American trauma, orthopedic, and thoracic surgeons on rib and sternal fracture repair. J Trauma 2009; 66: 875-9.

2) den Boer FC, Patka P, Bakker FC, et al. Current concepts of fracture healing, delayed unions, and nonunions. Osteo trauma care 2002; 10: 1-7.

3) Ergene G, Tulay CM, Anasiz H. Sternal fixation with nonspecific plate. Ann Thorac Cardiovasc Surg 2013; 19: 364-7.

4) Al-Qudah A. Operative treatment of sternal fractures. Asian Cardiovasc Thorac Ann 2006; 14: 399-401.

5) Snyder CW, Graham LA, Byers RE, et al. Primary sternal plating to prevent sternal wound complications after cardiac surgery: early experience and patterns of failure. Interact Cardiovasc Thorac Surg 2009; 9: 763-6.

6) Song DH, Lohman RF, Renucci JD, et al. Primary sternal plating in high-risk patients prevents mediastinitis. Eur J Cardiothorac Surg 2004; 26: 367-72.

7) Marco CA, Marco AP, Plewa MC, et al. The verbal numeric pain scale: effects of patient education on self-reports of pain. Acad Emerg Med 2006; 13: 853-9.

8) Dieselman JC. Comparison of alternative rigid sternal fixation techniques. A Thesis. Worcester Polytechnic Institute, USA, 2011; pp 11-31. 